\title{
Rabia Redouane, Femmes arabes et écritures francophones. Maghreb - Machrek
}

\section{Emanuela Cacchioli}

\section{(2) OpenEdition}

1 Journals

\section{Edizione digitale}

URL: http://journals.openedition.org/studifrancesi/4500

DOI: $10.4000 /$ studifrancesi. 4500

ISSN: 2421-5856

\section{Editore}

Rosenberg \& Sellier

\section{Edizione cartacea}

Data di pubblicazione: 1 settembre 2016

Paginazione: $375-376$

ISSN: 0039-2944

\section{Notizia bibliografica digitale}

Emanuela Cacchioli, «Rabia Redouane, Femmes arabes et écritures francophones. Maghreb - Machrek», Studi Francesi [Online], 179 (LX | II) | 2016, online dal 01 septembre 2016, consultato il 18 septembre 2020. URL : http://journals.openedition.org/studifrancesi/4500 ; DOI : https://doi.org/10.4000/ studifrancesi.4500

Questo documento è stato generato automaticamente il 18 settembre 2020.

\section{(c) (1)}

Studi Francesi è distribuita con Licenza Creative Commons Attribuzione - Non commerciale - Non opere derivate 4.0 Internazionale. 


\title{
Rabia Redouane, Femmes arabes et écritures francophones. Maghreb - Machrek
}

\author{
Emanuela Cacchioli
}

\section{NOTIZIA}

RABIA REDOUANE, Femmes arabes et écritures francophones. Maghreb - Machrek, Paris,

L'Harmattan, 2014, 308 pp.

1 Da sempre interessata alle opere di finzione di alcune autrici arabe e africane che scelgono il francese come lingua di espressione, Rabia REDOUANE ha dedicato proprio a questo tema la sua ultima monografia. La studiosa si è soffermata, in particolare, sulle tendenze comuni che caratterizzano la produzione letteraria di numerose autrici che hanno adottato la scrittura come mezzo di denuncia della società all'interno della quale vivono, ma anche come rivendicazione della loro esistenza in quanto esseri umani in grado di provare desideri e sentimenti al pari degli uomini. Redouane ha individuato un filo rosso che unisce la scrittura femminile di autrici che appartengono a due aree geograficamente distinte, ma che da sempre vengono accomunate a livello culturale. Le donne del Maghreb e del Machrek, infatti, affidano alle eroine dei loro romanzi i pensieri più intimi e la loro sete di rivendicazione. I loro personaggi diventano il simbolo di una generazione di donne che combattono l'ipocrisia e l'ingiustizia per ottenere una società priva di discriminazioni sociali e sessiste.

Il volume si suddivide in tre parti: le prime due sezioni sono dedicate al Machrek e al Maghreb, mentre l'ultima rappresenta una sorta di appendice in cui figurano brevi schede di lettura di romanzi scritti in francese da autrici di entrambe le aree geografiche (alcuni di questi sono già trattati più diffusamente nelle prime due sezioni).

Nella prima parte, «Écrivaines du Machrek» (pp. 19-122), ogni capitolo è riservato all'analisi delle modalità creative messe a punto da una diversa scrittrice. Il percorso si 
apre con la lettura di L'Excisée di Évelyne Accad, che, come suggerisce il titolo, descrive e denuncia la pratica delle mutilazioni genitali. Un atto che si pone come consacrazione del potere dell'uomo sull'universo femminile, ma soprattutto come conferma del fatto che le donne vivono in una situazione di ambiguità: rispetto a questo rito sono, al contempo, vittime, testimoni e complici. Di altro tenore è lo studio di L'Exil aux portes $d u$ paradis (1993) della scrittrice di origine egiziana Andrée Dahan. Il romanzo affronta il tema dello scontro tra l'Occidente e i paesi in via di sviluppo e dello sfruttamento dei ricchi ai danni dei poveri. Lo scenario sociale appare diviso tra l'abbondanza e il lusso dei villaggi vacanza e la situazione di miseria e di privazione della popolazione locale. Se gli investimenti dell'Occidente legati al turismo appaiono come una risorsa fondamentale per l'economia, essi generano comunque un ciclo vizioso dal quale solo pochi intermediari locali traggono reale beneficio. La studiosa prosegue la sua carrellata con un articolo su Sept pierres pour la femme adultère di Vénus Khoury-Ghata che tratta di un altro rito barbaro legato alla tradizione: la lapidazione di una donna adultera. Con questo romanzo la scrittrice denuncia la condizione di disumanità a cui viene ridotto l'essere umano che subisce tale destino. L'ultimo testo della prima parte è dedicato a Mariée à Paris. Répudiée à Beyrouth di Ezza Agha Malak. Al centro dell'opera troviamo tutte le sfaccettature psicologiche dei problemi di coppia, ma anche i codici di comportamento sociali e religiosi che una donna deve rispettare nel contesto libanese. La critica dell'autrice è dunque rivolta ai dettami imposti dalla tradizione ancestrale e dalla religione più intransigente, ma il testo vuole essere anche portatore di un confronto tra la cultura libanese e francese che possono anche trovare una forma di incontro e di comprensione reciproca.

4 La seconda sezione, «Écrivaines du Maghreb» (pp. 125-265), si apre con tre studi dedicati ad alcuni romanzi di Malika Mokkedem. Il primo si sofferma su una riflessione linguistica: nei romanzi Le Siècle des sauterelles et L'Interdite, la scrittrice algerina opta per una trasposizione dell'arabo all'interno di un tessuto testuale francese per rendere conto della sua complessa identità plurima. I suoi testi sono impregnati di riferimenti all'antichità e al patrimonio culturale arabo che possono essere resi solo se si ricorre a tale idioma. Numerosi sono quindi gli espedienti che deve mettere in pratica per permettere la fruizione del testo anche al lettore che non possiede tale conoscenza. Il secondo articolo affronta, invece, la presa di coscienza della condizione di esule che la scrittrice tratta in N'zid. È grazie alla distanza fisica dall'Algeria che Mokkeddem può riformulare il rapporto con la terra d'origine e riscoprirne la bellezza. Nel romanzo Mes hommes la scrittrice ripercorre la metamorfosi della sua identità dovuta al conflitto con la figura paterna e alla ricerca di amore, inteso come rispetto dell'altro e condivisione. Si tratta di un testo autobiografico in cui l'autrice tende al raggiungimento della felicità, della libertà e dell'emancipazione. Al centro del quarto articolo, Redouane pone Rêves d'enfance di Fatima Mernissi che ci offre uno sguardo sull'harem, inteso come luogo domestico di chiusura paragonato a una prigione, nel quale le uniche forme di trasgressione per accedere all'esterno sono la radio e un piccolo terrazzo senza protezioni. La lotta per l'emancipazione è il tema del romanzo Jeux de rubans di Emna Belhaj Yahia: il punto di vista di una donna cresciuta da una madre coraggiosa in un ambiente libero da costrizioni si scontra con quello della fidanzata del figlio che predilige la tradizione e la manifesta attraverso la scelta del velo e dell'abito lungo. Segue una lettura di Fille de harki di Fatima Besnaci-Lancou, testo in cui l'autrice algerina offre una testimonianza storica, politica e sociale delle sofferenze provate dagli harki, ossia gli Algerini che hanno combattuto la guerra d'Algeria al fianco dei 
francesi. A conclusione della sezione è stata inserita una lettura di Louve musulmane di Amale El Atrassi. Anche in questo caso si tratta di una denuncia degli abusi subiti in famiglia durante l'adolescenza da parte della protagonista e della volontà di affermare la propria libertà e la propria esistenza.

5 Chiude il volume la terza parte (pp. 267-300), nella quale troviamo tredici schede di lettura critica piuttosto sintetiche di alcuni romanzi di Évelyne Accad, Ezza Agha Malak, Soumya Ammar Khodja, Latifa Ben Mansour, Lamia Bereksi Meddahi, Maïssa Bey, Yasmine Char, Leila Marouane, Malika Mokkeddem e Karima Perrin.

La specificità dell'opera consiste nella volontà della studiosa di unire le due aree geografiche e culturali per mezzo di una riflessione sulla scrittura femminile. Il risultato è un volume in grado di arricchire al tempo stesso gli studi critici di letteratura comparata e quelli sociologici che mettono al centro la condizione della donna nel mondo arabo. La debolezza di questo volume risiede invece nelle modalità attraverso le quali queste riflessioni vengono condotte. Gli aspetti relativi allo studio sociologico e alla comparazione dei due ambiti letterari vengono presentati nell'introduzione dell'opera, ma non sono mai sistematizzati in una riflessione teorica compiuta. È il lettore che deve lasciarsi guidare dalle suggestioni offerte nelle analisi dei singoli testi e operare autonomamente il sunto delle due prospettive adottate dalla studiosa e tracciare così la "passerelle entre deux régions sociales et culturelles convergentes et en même temps différentes dans cet espace géographique qu'est le Monde arabe d'aujourd'hui» (p. 17). 